\title{
Descripción del estado nutricional de los pacientes de una unidad de diálisis mediante el uso de la escala "Malnutrition Inflamation Score"
}

\author{
Elvira Carrascal Sonia*, Colomer Codinachs Marta*, Pérez Oller Laureano**, Chirveches Pérez Emma***, \\ Puigoriol Juvanteny Emma****, Pajares Requena Dolores*, Rusiñol Camps Carme*, Prat Canals Rosa \\ María*, Castells Prat Montse*****, Roquet Bohils Marta*, Serrano Vilalta Maite*
}

*Diplomada en Enfermería Unidad de Nefrología, * * Médico Adjunto de la Unidad de Nefrología, ** *Diplomada en enfermería de la Unidad Clínico-epidemiológica, ****Diplomada en Estadística de la Unidad Clínicoepidemiológica, ${ }^{* * * * *}$ Dietista-nutricionista del Servicio de Endocrinología y Nutrición del "Consorci Hospitalari de Vic". Barcelona

\section{Resumen}

\section{Introducción}

La desnutrición es un problema de salud muy frecuente en la población con insuficiencia renal crónica en diálisis. Dada la alta prevalencia de malnutrición en los pacientes en diálisis y su repercusión sobre la morbi-mortalidad de los pacientes es preciso instaurar un adecuado seguimiento de los aspectos nutricionales, para detectar precozmente a pacientes en riesgo o con déficit nutricionales y realizar una intervención nutricional precoz que consiga revertir la situación.

\section{Objetivos}

- Describir el estado nutricional de los pacientes atendidos en la unidad de diálisis del servicio de nefrología del Consorcio Hospitalario de Vic, según la escala "Malnutrition Inflamation Score" y durante un período de 2 años.

- Describir las características sociodemográficas y clínicas de los pacientes en diálisis del Consorcio Hospitalario de Vic que han sido sometidos a una valoración de su estado nutricional, mediante la escala "Malnutrition Inflamation Score".

- Analizar si factores como el género, la edad, el tipo de tratamiento dialítico, la presencia de comorbilidad, el tipo de vía de acceso de hemodiálisis, el tiempo en tratamiento dialítico, el

Correspondencia:

Sonia Elvira Carrascal

Unidad Nefrológica. Consorcio Hospitalario de Vic

$\mathrm{C} /$. Francesc Pla "el vigatá" $\mathrm{s} / \mathrm{n}$

08500, Vic, Barcelona

E-mail: selvira@chv.cat número de ingresos o el total de días de ingreso están relacionados con el estado nutricional de los pacientes en diálisis del Consorcio Hospitalario de Vic, según las puntuaciones obtenidas de la escala "Malnutrition Inflamation Score".

\section{Material y métodos}

Estudio transversal prospectivo. Realizado en la Unidad Nefrológica del Consorcio Hospitalario de Vic. Se estudiaron los enfermos diagnosticados de enfermedad renal crónica sometidos a diálisis y se incluyeron los pacientes en diálisis y con un historial de tratamiento de más de tres meses. El estudio se realizó de Enero del 2009 hasta Diciembre de 2010. Se analizaron variables sociodemográficas (edad, género, tipo de tratamiento dialítico, origen de la nefropatía, existencia de historial de comorbilidad, vía de acceso para la hemodiálisis, situación tratamiento dialítico, causa finalización tratamiento dialítico), el estado nutricional según escala "Malnutrition Inflamation Score" y e/ consumo de recursos (número de ingresos anuales, total de días de ingresos anuales, tiempo de tratamiento en diálisis).Los datos se obtuvieron de los registros informatizados del servicio de nefrología. Para el análisis estadístico se utilizó el programa SPSS v.18.0.

\section{Resultados}

Se analizaron 130 pacientes que correspondieron al total de los evaluados según el estado nutricional, mediante la escala "Malnutrition Inflamation Score" durante los años 2009 y 2010. De ellos eran hombres $79(60,8 \%)$ y mujeres $51(39,2 \%)$, la edad media fue de $64,76 \pm 14,27$ años y estaban desnutridos el $18,5 \%$ de los pacientes. Del total de 130 pacientes analizados se obtuvieron 399 valores de la escala "Malnutrition Inflamation Score". De 69 $(53,1 \%)$ de ellos, se disponía de 4 registros de la es- 
cala "Malnutrition Inflamation Score", $18(13,8 \%)$ de 3 registros y $26(20,0 \%)$ de 2 y $17(13,1 \%)$ solo tenía un registro.. Se observó que $353(88,5 \%)$ registros puntuaban como bien nutridos y 46 ( $11,5 \%)$ desnutridos. Las puntuaciones medias de la escala "Malnutrition Inflamation Score" según el número de registros (del primero al cuarto) fueron de: 6,29 $\pm 3,65,6,06 \pm 2,91,6,38 \pm 3,13$ y $7,96 \pm 3,50$ respectivamente pudiéndose observar diferencias estadísticamente significativas $(p=0,001)$.Se analizó la evolución nutricional de 113 pacientes $(86,9 \%)$ que eran aquellos que se disponía de más de un registro de la escala "Malnutrition Inflamation Score". Se tuvieron en cuenta los valores "Malnutrition Inflamation Score" del primero y último registro. La puntuación media de los primeros registros era de $5,96 \pm 3,34$ frente a la media de los finales que fue 7,50 $\pm 3,62$ siendo estas diferencias estadísticamente significativas $(p<0,001)$.

\section{Conclusiones}

- Destacar la importancia de conocer el estado nutricional de los pacientes con insuficiencia renal crónica en tratamiento de hemodiálisis y diálisis peritoneal por el riesgo que sufren éstos de presentar morbimortalidad asociada.

- La escala "Malnutrition Inflamation Score" es un buen instrumento de valoración del estado nutricional, que facilita el cuidado de los pacientes en diálisis.

- El tiempo en tratamiento sustitutivo y la presencia de comorbilidad son dos de los principales aspectos a tener en cuenta a la hora de controlar el estado nutricional de los pacientes con enfermedad renal crónica.

- Hay que continuar con la realización de estudios de aplicación de la escala "Malnutrition Inflamation Score" para valorar el estado nutricional y que el nivel de evidencia se modifique.

\section{PALABRAS CLAVE:}

- DESNUTRICIÓN

- MALNUTRICIÓN

- MIS "MALNUTRITION INFLAMATION SCORE"

- COMORBILIDAD

- ESTADO NUTRICIONAL

\section{Description of the nutritional state of patients in a dialysis unit using the Malnutrition Inflamma- tion Score}

\section{Abstract}

\section{Introduction}

Malnutrition is a very frequent health problem in the population with chronic kidney insufficiency receiving dialysis. Given the high prevalence of malnutrition in dialysis patients and its effect on morbimortality of patients, it is necessary to establish an adequate monitoring of nutritional aspects for early detection of patients at risk or with nutritional deficit and carry out an early nutritional intervention that will manage to reverse the situation.

\section{Objectives}

- To describe the nutritional state of patients attended at the dialysis unit of the nephrology service of the Consorcio Hospitalario de Vic, according to the Malnutrition Inflammation Score for a period of 2 years.

- To describe the socio-demographic and clinical features of dialysis patients in the Consorcio Hospitalario de Vic whose nutritional state was assessed using the Malnutrition Inflammation Score.

- To analyse whether factors such as gender, age, type of dialysis treatment, the presence of comorbility, the type of haemodialysis access, the time on dialysis, the number of hospitalizations or total number of days hospitalized are related to the nutritional state of dialysis patients at the Consorcio Hospitalario de Vic, according to the Malnutrition Inflammation Scores obtained.

\section{Material and methods}

Prospective transversal study. Carried out at the Nephrology Unit of the Consorcio Hospitalario de Vic. Patients diagnosed with chronic kidney disease and who undergo dialysis were studied, and dialysis patients with treatment records of more than three months were included. The study was carried out from January 2009 to December 2010. Sociodemographic variables (age, gender, type of dialysis treatment, origin of the renal pathology, existence of history of comorbility, access used for haemodialysis, situation of dialysis treatment, cause for end 
of dialysis treatment), the nutritional state according to the Malnutrition Inflammation Score and consumption of resources (number of hospitalizations per year, total number of days hospitalized per year, time on dialysis) were analysed. The data were obtained from the computerized records of the nephrology service. The SPSS v.18.0. programme was used for the statistical analysis.

\section{Results}

130 patients were analysed, which corresponded to the total number of patients assessed according to nutritional state using the Malnutrition Inflammation Score during 2009 and 2010. Of these, $79(60.8 \%)$ were men and $51(39.2 \%)$ were women, the average age was $64.76 \pm 14.27$ years, and $18.5 \%$ of the patients were malnourished. Of the total of 130 patients analysed, 399 Malnutrition Inflammation Score values were obtained. From $69(53.1 \%)$ of them, there were 4 scores, from $18(13.8 \%)$ there were 3 scores and for $26(20,0 \%)$ there were 2 and for 17 $(13.1 \%)$ there was only one. It was observed that $353(88.5 \%)$ of the scores were classified as well-nourished and $46(11.5 \%)$ as malnourished. The mean scores on the Malnutrition Inflammation Score scale according to the number of records (from the first to the fourth) were: $6.29 \pm 3.65,6.06 \pm 2.91,6.38 \pm 3.13$ and 7.96 \pm 3.50 respectively, with statistically significant differences being observed $(p=0.001)$. The nutritional evolution of 113 patients $(86.9 \%)$ was analysed, who were those who had more than one record of Malnutrition Inflammation Score. The scores of the first and last record were taken into account. The average score for the first records was $5.96 \pm 3.34$ compared to the average for the final scores which was $7.50 \pm 3.62$, these differences being statistically significant $(p<0.001)$.

\section{Conclusions}

- We would highlight the importance of knowing the nutritional state of chronic kidney insufficiency patients receiving haemodialysis and peritoneal dialysis treatment due to the risk of presenting associated morbi-mortality.

- The Malnutrition Inflammation Score is a good instrument for assessing nutritional state, facilitating the care of dialysis patients.
- The time on replacement therapy and the presence of comorbility are two of the main aspects to take into consideration when controlling the nutritional state of patients with chronic kidney disease.

- Studies should continue to be carried out on the application of the Malnutrition Inflammation Score in assessing the nutritional state and the level of evidence should be modified.

\section{KEY WORDS:}

- UNDERNUTRITION

- MALNUTRITION

- MIS (MALNUTRITION INFLAMMATION SCORE)

- COMORBILITY

- NUTRITIONAL STATE

\section{Introducción}

Según la Organización Mundial de la Salud la nutrición es la ingesta de alimentos en relación con las necesidades dietéticas del organismo. Una buena nutrición, que comporta una dieta suficiente y equilibrada combinada con el ejercicio físico regular es un elemento fundamental de la buena salud ${ }^{1}$.

La desnutrición está considerada como una enfermedad con entidad propia, que requiere un abordaje específico para su diagnóstico y tratamiento. Pero su diagnóstico es complejo puesto que no se dispone de un parámetro sencillo y reproducible para definirla con precisión ${ }^{2}$. El concepto de desnutrición hace referencia, no solamente a deficiencias en los marcadores bioquímicos, antropométricos y/o de las reservas de nutrientes, o de composición corporal, sino que además engloba complicaciones a las que es susceptible un sujeto que padece un déficit de reservas calórico-proteicas ${ }^{3}$.

La desnutrición es un problema de salud muy frecuente en la población con insuficiencia renal crónica en diáli$\mathrm{sis}^{4}$. Las causas de desnutrición en estos pacientes son multifactoriales, ya que comprenden alteraciones del metabolismo proteico y energético, alteraciones hormonales e ingesta alimentaria deficiente, sobre todo a causa de la anorexia y de las náuseas y vómitos relacionados con el estado de toxicidad urémica. Varias enfermedades concomitantes, como la diabetes mellitus 
y la enfermedad vascular difusa, así como las afecciones intercurrentes (pericarditis, infecciones, insuficiencia cardíaca congestiva), que pueden contribuir a la desnutrición ${ }^{5}$.

Es fundamental, definir la situación nutricional de los pacientes en diálisis y su evaluación a fin de identificar los pacientes desnutridos o en riesgo, para ayudar a corregir esta situación ${ }^{5}$. Con el fin de establecer una metodología en la valoración del estado nutricional, seguimiento e intervención en los pacientes en diálisis, la Sociedad Española de Diálisis y Transplante a través de un grupo de expertos elaboraron las recomendaciones que se exponen a continuación:

- La valoración nutricional debe sustentarse en el análisis de una combinación de variables, que evalúan aspectos distintos y complementarios del estado nutricional. No hay ningún parámetro que individualmente sea capaz de establecer el estado nutricional global y varios son los que están independientemente relacionados con la morbimortalidad.

- Es recomendable disponer de un dietista-nutricionista que realice la historia clínica nutricional, para así valorar íntegramente el estado nutricional del paciente, detectar hábitos nutricionales de riesgo y realizar el seguimiento y control evolutivo.

- La exploración física debe realizarse siempre tras la diálisis y estando el paciente en su peso seco. La antropometría debe incluir el porcentaje del peso corporal habitual y del peso estándar, el índice de masa corporal, los pliegues cutáneos y la circunferencia del brazo.

- El panel de datos analíticos se realizará siempre antes de la diálisis del día mitad de semana. En diálisis peritoneal se realizará indistintamente cualquier día, salvo cambios de pauta en fin de semana.

- El método ideal de análisis de la composición corporal es el DEXA, pero su coste y disponibilidad, limitan su uso a estudios de investigación. La antropometría es una alternativa rápida, precisa y reproductible con una alta correlación con los datos obtenidos por DEXA. La bioimpedancia (BIA) es un método fundamentalmente válido para valorar el estado de hidratación, estando el BIA vector (BIVA) pendiente de validación clínica que confirme su utilidad en el análisis de la composición corporal y nutrición de los pacientes en diálisis ${ }^{6}$.
Dada la alta prevalencia de malnutrición en los pacientes en diálisis y su repercusión sobre la morbi-mortalidad es preciso instaurar un adecuado seguimiento de los aspectos nutricionales, para detectar precozmente a pacientes en riesgo o con déficit nutricionales y realizar una intervención precoz que consiga revertir la situación. Por ello en la Unidad de Nefrología del Consorcio Hospitalario de Vic, en el año 2001 se creó un equipo interdisciplinar compuesto por una enfermera y un nefrólogo con formación específica sobre nutrición y la dietista del centro. Se elaboró un libro informativo y se realizaron diferentes reuniones informativas dirigidas a los enfermos y sus familiares con el fin de exponerles unas pautas básicas de alimentación. En el año 2003, se editó una versión dirigida a los pacientes en hemodiálisis afectos de diabetes. A partir del 2004 se inició la individualización de la información sobre la dieta. En el 2008, se editó otra versión dirigida a los pacientes que presentaban niveles de fósforo alto.

Finalmente en el 2009-2010 se empezó a trabajar con la escala "Malnutrition Inflamation Score" (MIS). Esta se pasa a todos los pacientes que llevan más de 3 meses en diálisis. Se trata de un test cuantitativo que valora globalmente la nutrición y la inflamación. Valora en total 10 variables, con 4 niveles de severidad que varían de 0 (normal) a 3 (muy severo). La suma de todas las puntuaciones determina el grado de nutrición del paciente que puede variar desde 0 a 3055.7.

Se elaboró un plan de acción de valoración nutricional que contempla una entrevista dietética y la realización de la escala MIS $^{5-7}$ a partir de los 3 primeros meses de iniciar el tratamiento, así como la valoración de la escala MIS de forma programada cada 6 meses $y$, en caso de procesos intercurrentes 0 otras situaciones que hagan sospechar riesgo de desnutrición del paciente, se valora y repite la entrevista dietética y se adelanta la realización de la escala MIS. En la actualidad (2011) se está elaborando un protocolo de actuación sobre los pacientes en que se detecta una situación o riesgo de desnutrición.

\section{Objetivo}

\section{Principal:}

- Describir el estado nutricional de los pacientes atendidos en la unidad de diálisis del servicio de nefrología del Consorcio Hospitalario de Vic, según la escala MIS y durante un período de 2 años. 


\section{Secundarios:}

- Comparar las puntuaciones iniciales MIS ( $1^{a}$ escala obtenida) con las finales (última escala obtenida) de los pacientes atendidos en la unidad de diálisis del servicio de nefrología del Consorcio Hospitalario de Vic.

- Describir las características sociodemográficas y clínicas de los pacientes en diálisis del Consorcio Hospitalario de Vic que han sido sometidos a una valoración de su estado nutricional, mediante la escala MIS.

- Analizar si factores como el género, la edad, el tipo de tratamiento dialítico, la presencia de comorbilidad, el tipo de vía de acceso hemodiálisis, el tiempo en tratamiento dialítico, el número de ingresos o el total de días de ingreso están relacionados con el estado nutricional de los pacientes en diálisis del Consorcio Hospitalario de Vic, según las puntuaciones obtenidas de la escala MIS.

\section{Material y métodos}

Tipo de estudio: transversal prospectivo.

Centro de estudio: Unidad Nefrológica del Consorcio Hospitalario de Vic.

Población de estudio: pacientes diagnosticados de Enfermedad Renal Crónica sometidos a diálisis.

Criterios de inclusión: pacientes en diálisis y con un historial de tratamiento de más de 3 meses.

Criterios de exclusión: pacientes en diálisis y con un historial de tratamiento de menos de 3 meses.

Periodo de estudio: desde Enero del 2009 a Diciembre 2010 (2 años).

\section{Variables de estudio:}

- Estado nutricional: bien nutrido (<10 escala MIS), mal nutrido ( $>10$ escala MIS). Para concretar el estado nutricional se tuvo en cuenta el valor de la última escala recogida durante el período de estudio de cada paciente. La puntuación de la escala MIS $^{5-7}$ se obtiene de las puntuaciones de una escala Likert (0-3 ) que tiene en cuenta los siguientes parámetros:

1. La historia médica del paciente teniendo en cuenta el cambio de peso seco al final de la diálisis (cambio total en los últimos 3-6 meses), consumo dietético, síntomas gastrointestinales (GI), capacidad funcional (daño funcional relacionado con la nutrición), co-morbilidad (incluyendo los años en diálisis).

2. Examen físico: valoración de disminución de las reservas de grasas mediante la medida del pliegue tricipital (PTC) y de signos de pérdida muscular (sien, clavícula, costillas, cuádriceps, rodilla intraósea).

3. Índice de masa corporal $\left(\mathrm{IMC}=\mathrm{kg} / \mathrm{m}^{2}\right)$.

4. Parámetros de laboratorio: albúmina en suero, transferrina sérica.

- Variables sociodemográficas y clínicas: edad, género (hombre, mujer), tipo de tratamiento dialítico (hemodiálisis, diálisis peritoneal), origen de la nefropatía (diabetes, poliquísticos, vasculitis, glomerulonefritis, insuficiencia renal crónica no filiada y HTA), existencia de historial de comorbilidad (sí, no), vía de acceso para la hemodiálisis (catéter, FAVI), situación tratamiento dialítico (activo, finalizado), causa finalización tratamiento dialítico (exitus, trasplante, traslado a otro centro).

- Consumo de recursos: número de ingresos, total de días ingreso, tiempo de tratamiento en diálisis.

\section{Recogida de los datos:}

Los datos fueron obtenidos de los registros informatizados del servicio de nefrología. En ellos, semestralmente y de manera habitual se registran las puntuaciones de la valoración completa de malnutrición-inflamación según la escala MIS.

En nuestro caso, para medir el PTC se requiere de un plicómetro, una cinta métrica y una enfermera por turno previamente entrenada ya que, según las guías DOQI ${ }^{8-}$ 11, se debe realizar al final de la sesión de diálisis de mitad de semana y por la misma persona para así aumentar la fiabilidad de los resultados. Una vez obtenido el resultado de la medida se compara con las tablas de Alastrúe $e^{12}$ por edad y sexo y se obtiene el percentil que indica el grado de desnutrición. Para calcular el índice de Masa Corporal, se requiere disponer "del peso seco actual" y "la medida de la talla anual". Y por último, los parámetros de laboratorio utilizados para el cálculo del MIS corresponden a los que coinciden con el mes en el que se valora el MIS y se cursan durante la diálisis de mitad de semana. 


\section{Análisis estadístico:}

Para el análisis estadístico se utilizó el programa SPSS v.18.0. Para la obtención de los resultados se ha realizado un análisis descriptivo.

Las variables cualitativas se expresaron como número de casos y porcentaje, y se compararon mediante el test de la ${ }^{2}$ o la prueba exacta de Fisher cuando no se cumplían los criterios de aplicabilidad de la primera. Las variables cuantitativas se describieron mediante la media \pm desviación estándar o la mediana y los percentiles 25 y 75 , en el caso de que las distribuciones fueran asimétricas. La comparación de estas variables se llevó a cabo mediante la prueba de la $t$ de Student o la prueba no paramétrica de la $U$ de Mann-Whitney en el caso de variables con distribución no normal. Para analizar la evolución (inicial-final) de las puntuaciones de la escala MIS se utilizó la prueba de T-test de pruebas relacionadas.

\section{Resultados}

Se analizaron 130 pacientes que correspondieron al total de los evaluados según el estado nutricional, mediante la escala MIS durante los años 2009 y 2010. De ellos eran hombres $79(60,8 \%)$ y mujeres $51(39,2 \%)$. La edad media fue de 64,76 $\pm 14,27$ años, con un mínimo de 33 años y un máximo de 88.75 años. Presentaban desnutrición el $18,5 \%$ de los pacientes.

Estaban diagnosticados de insuficiencia renal crónica de etiología no filiada $73(56,2 \%)$, nefropatía diabética $16(12,3 \%)$, poliquistosis renal $12(9,2 \%)$, pielonefritis crónica $10(7,7 \%)$, enfermedad vascular renal $10(7,7 \%)$, glomerulonefritis $2(1,5 \%)$ y otras causas $7(5,4 \%)$ pacientes respectivamente. Presentaban historial de comorbilidad 56(43,1\%).

Respecto al tratamiento, estaban en hemodiálisis $114(87,7 \%)$ y en diálisis peritoneal $16(12,3 \%)$. De los pacientes en hemodiálisis, eran portadores de FAVI $91(79,8 \%)$ y de catéter $23(20,2 \%)$.

Los pacientes que durante todo el período de estudio estuvieron en diálisis presentaron una mediana de 30,58 meses de tratamiento con unos percentiles 25 y 75 de 19,5 y 58,62 respectivamente, en cambio los que dejaron de ser tratados presentaron una mediana de 45,43 meses con unos percentiles de 23,3 y 63,9 respectivamente. Los pacientes que abandonaron el tratamiento fueron $40(30,8 \%)$ y los motivos: exitus 24
$(60,0 \%)$, trasplante $14(35,0 \%)$ y traslado a otro centro $2(5,0 \%)$.

Durante el estudio ingresaron en el hospital $96(73,8 \%)$ pacientes con una mediana y unos percentiles de 2,00 $(1,00-4,00)$ ingresos por paciente, siendo la mediana y los percentiles del total de días de ingreso de 8,00 $(3,25-22,75)$.

Del total de 130 pacientes analizados se obtuvieron 399 valores de la escala MIS. De $69(53,1 \%)$ de ellos, se disponía de 4 registros de la escalas MIS, 18 (13,8\%) de 3 registros y $26(20,0 \%)$ de 2 y $17(13,1 \%)$ solo tenía un registro. Se observó que $353(88,5 \%)$ registros puntuaban como bien nutridos y $46(11,5 \%)$ desnutridos. Las puntuaciones medias de la MIS según el número de registros (del primero al cuarto) fueron de: $6,29 \pm 3,65,6,06 \pm 2,91,6,38 \pm 3,13$ y 7,96 $\pm 3,50$ respectivamente pudiéndose observar diferencias estadísticamente significativas $(p=0,001)$.

Se analizó la evolución nutricional de 113 pacientes $(86,9 \%)$ que eran aquellos que se disponía de más de un registro de la escala MIS. Se tuvieron en cuenta los valores MIS del primero y último registro. La puntuación

\begin{tabular}{|c|c|c|c|}
\hline & $\begin{array}{c}\text { Bien nutridos } \\
\begin{array}{c}(\mathrm{N}=106) \\
81,5 \%\end{array}\end{array}$ & $\begin{array}{c}\text { Mal nutridos } \\
\begin{array}{c}(\mathrm{N}=24) \\
18,5 \%\end{array}\end{array}$ & \multirow[t]{2}{*}{ p-valor } \\
\hline & n (\%) & n (\%) & \\
\hline $\begin{array}{l}\text { Género } \\
\text { Hombre } \\
\text { Mujer }\end{array}$ & $\begin{array}{l}68(86,1 \%) \\
38(74,5 \%)\end{array}$ & $\begin{array}{l}11(13,9 \%) \\
13(25,5 \%)\end{array}$ & 0,097 \\
\hline Edad, media (DE) & $64,51(13,51)$ & $65,87(17,50)$ & 0,675 \\
\hline $\begin{array}{l}\text { Tipo de tratamiento } \\
\text { dialítico } \\
\text { Hemodiálisis } \\
\text { Diálisis Peritoneal }\end{array}$ & $\begin{array}{l}91(79,8 \%) \\
15(93,8 \%)\end{array}$ & $\begin{array}{c}23(20,2 \%) \\
1(6,3 \%)\end{array}$ & 0,179 \\
\hline $\begin{array}{l}\text { Presencia de } \\
\text { Comorbilidad } \\
\text { Sí } \\
\text { No }\end{array}$ & $\begin{array}{l}39(69,6 \%) \\
67(90,5 \%)\end{array}$ & $\begin{array}{c}17(30,4 \%) \\
7(9,5 \%)\end{array}$ & $0,002 *$ \\
\hline $\begin{array}{l}\text { Situación } \\
\text { tratamiento dialítico } \\
\text { Finalizado } \\
\text { Activo }\end{array}$ & $\begin{array}{l}33(73,3 \%) \\
73(85,9 \%)\end{array}$ & $\begin{array}{l}12(26,7 \%) \\
12(14,1 \%)\end{array}$ & 0,079 \\
\hline $\begin{array}{l}\text { Vía de acceso } \\
\text { Hemodiálisis } \\
\text { Catéter } \\
\text { FAVI }\end{array}$ & $\begin{array}{l}19(82,6 \%) \\
72(79,1 \%)\end{array}$ & $\begin{array}{c}4(17,4 \%) \\
19(20,9 \%)\end{array}$ & 0,710 \\
\hline
\end{tabular}

Tabla 1. Descripción sociodemográfica y clínica de los pacientes en diálisis según su estado de nutrición 


\begin{tabular}{|l|c|c|c|c|}
\hline & \multicolumn{3}{|c|}{ Bien nutridos } & \multicolumn{2}{c|}{ Mal nutridos } \\
\cline { 2 - 6 } & $\mathbf{n}$ & Mediana (P25-P75) & $\mathbf{n}$ & Mediana (P25-P75) \\
\hline Tiempo en tratamiento & 106 & $30,9(18,9-55,2)$ & 24 & $59,6(40,9-110,0)$ \\
\hline Número total de ingresos & 77 & $2,0(1,0-4,0)$ & 19 & $3,0(1,0-4,0)$ \\
\hline Número total de días de ingreso & 77 & $8,0(3,0-20,0)$ & 19 & $12,0(5,0-36,0)$ \\
\hline
\end{tabular}

Tabla 2. Consumo de recursos de los pacientes en diálisis según su estado de nutrición

media de los primeros registros era de 5,96 $\pm 3,34$ frente a la media de los finales que fue $7,50 \pm 3,62$ siendo estas diferencias estadísticamente significativas $(p<0,001)$.

Los resultados obtenidos respecto a la relación existente entre estar bien o mal nutridos y las variables sociodemográficas, clínicas y consumo de recursos analizados se muestran en la tabla $\mathbf{1}$, tabla 2. En ellas puede observarse que existe una diferencia estadísticamente significativa $(p=0,002)$ entre la presencia de comorbilidad y el estado nutricional y también entre éste ultimo y el tiempo en tratamiento de diálisis $(p<0,001)$.

En la tabla 3 se muestra la relación existente entre estar bien o mal nutridos y el consumo de recursos de los pacientes con 4 registros de la escala MIS (pacientes que estuvieron en diálisis durante todo el período de estudio), las diferencias estadísticamente observadas son las mismas que se observaron en la tabla 2.

\begin{tabular}{|l|c|c|c|c|}
\hline & \multicolumn{2}{|c|}{ Bien nutridos } & \multicolumn{2}{|c|}{ Mal nutridos } \\
\cline { 2 - 6 } & $\mathbf{n}$ & Mediana (P25-P75) & $\mathbf{n}$ & Mediana (P25-P75) \\
\hline Tiempo en tratamiento & 53 & $37,33(26,4-66,6)$ & 16 & $57,87(41,4-110,0)$ \\
\hline Número total de ingresos & 37 & $2,0(1,0-3,5)$ & 13 & $2,0(1,0-3,5)$ \\
\hline Número total de días de ingreso & 37 & $6,0(3,0-15,5)$ & 13 & $7,0(2,0-23,5)$ \\
\hline
\end{tabular}

Tabla 3. Consumo de recursos de los pacientes que estuvieron en diálisis durante todo el período de estudio según su estado de nutrición

\section{Discusión}

La prevalencia de malnutrición en los pacientes de diálisis, varía según diferentes autores, debido a la existencia de diferentes métodos de valoración y a diferentes criterios diagnósticos ${ }^{6}$.

Aunque existen otras escalas de valoración nutricional, en este estudio se ha utilizado la escala MIS puesto que se consideró que sería la más adecuada para evaluar los pacientes tratados en diálisis, ya que tiene en cuenta varios parámetros (de historia clínica, exploración física y datos analíticos) y la suma de ellos se consideró que podía evaluar con más exactitud el estado nutricional.

Con el uso de la escala MIS en este estudio se ha podido concretar que los enfermos que presentaban más comorbilidades y que llevaban más tiempo de tratamiento dialítico presentaban más prevalencia de malnutrición hecho que influye negativamente en la morbimortalidad de estos pacientes. Se ha podido comprobar cómo la puntuación del MIS va en aumento en relación al incremento de tiempo en tratamiento dialítico y por ello se puede prever que a más tiempo de diálisis más riesgo de desnutrición.

Como profesionales de enfermería, creemos que la sesión de diálisis es un buen momento para realizar la valoración del estado nutricional puesto que se produce un contacto frecuente y constante que se mantiene cada día con los pacientes ${ }^{8}$.

Se ha podido constatar que pasar la escala MIS es un proceso rápido, económico, fácil y práctico. No obstante es importante unificar los criterios de valoración de la escala MIS a seguir por el equipo de enfermería, para que los resultados sean más fiables.

Para finalizar se destaca el papel de la enfermera en relación a la educación dietética del enfermo que con- 
tribuye a la prevención, detección y corrección de los posibles factores que influyen en el estado nutricional ${ }^{11}$. Es importante individualizar la dieta según los parámetros analíticos y plantear dietas libres en aquellos enfermos que analíticamente lo permitan. Cabe resaltar, la importancia de aumentar la ingesta proteica mediante aportes proteicos si los pacientes lo necesitan.

\section{Conclusiones}

Destacar la importancia de conocer el estado nutricional de los pacientes con insuficiencia renal crónica en tratamiento de hemodiálisis y diálisis peritoneal por el riesgo que sufren éstos de presentar morbimortalidad asociada. La escala MIS es un buen instrumento de valoración del estado nutricional, que facilita el cuidado de los pacientes en diálisis.

El tiempo en tratamiento sustitutivo y la presencia de comorbilidad son dos de los principales aspectos a tener en cuenta a la hora de controlar el estado nutricional de los pacientes con enfermedad renal crónica.

Hay que continuar con la realización de estudios de aplicación de la escala MIS para valorar el estado nutricional y que el nivel de evidencia se modifique.

Recibido: 20 Noviembre 2012

Revisado: 30 Noviembre 2012

Modificado: 31 Enero 2013

Aceptado: 2 Febrero 2013

\section{Bibliografía}

1. Organización Mundial de la Salud. [acceso 26 de Abril 2011]. Disponible en: http://www.who.int/ topics/nutrition/es/.

2. Valoración del estado nutricional de pacientes mayores de 65 años en tratamiento sustitutivo en una unidad de diálisis. J.Gómez Macías. Unidad de Diálisis. Fundación Jiménez Díaz. Madrid. Rev. Soc. Esp. Enferm. Nefrol.2006;(9) 2:12-18.

3. Aspectos nutricionales en hemodiálisis. Victor Lorenzo, Margarita Rufino y Marisa Martín.
Cap.24 Tratado de hemodiálisis. Fernando Valderrábano. Editorial JIMS, S,L 1999.

4. J. M. Manzano Angua. Valoración antropométrica de la población renal crónica estable en hemodiálisis en la provincia de Sevilla. Rev. Soc. Esp. Enferm. Nefrol.2006;(9) 3:66-73.

5. Riella MC, Martins C. Nutrición y Riñón.1 ${ }^{a}$.ed. Madrid: Panaamericana;2006.p.77-87, 122-142, 307-368.

6. Huarte-Loza et al. Nutrición en pacientes en diálisis. Consenso SEDYT. Dial Traspl.2006;27(4):13861.

7. Kamyar Kalantar-Zadeh, MD, Joel D. Kopple, MD, Gladys Block, PhD, and Michael H. Humphreys, MD. A Malnutrition-Inflammation Score Is Correlated With Morbidity and Mortality in Maintenance Hemodialysis Patients. Disponible en: http://www. neprology.

8. Manzano Angua JM, Nieto Granados MD, Sánchez Cornejo MC. Parámetros antropométricos más idóneos para valorar el estado nutricional de los pacientes con insuficiencia renal crónica, tratados con hemodiálisis en centros periféricos. Rev. Soc. Esp. Enferm. Nefrol. 2003;(6)3:6-15.

9. Valoración nutricional de enfermería de los pacientes tratados con hemodiálisis en un centro periférico. JM. Manzano Angua, MD.Nieto Granados, MC.Sánchez Cornejo. Centro de diálisis CAMEXS/A.Bellavista.Sevilla. Rev. Soc. Esp. Enferm. Nefrol. 2004; (7) 1:10-18.

10. Guías K/DOQI nutrition in chronic renal failure, Am.J.Kidney.june 2000.

11. Manzano Angua JM, Nieto Granados MD. Influencia de los criterios de clasificación sobre la valoración nutricional de enfermería mediante parámetros antropométricos. Rev. Soc. Esp. Enferm. Nefrol. 2005;(8)1:6-12.

12. Alastrué Vidal et al. Nuevas normas y consejos en la valoración de los parámetros antropométricos en nuestra población: índice adiposo-muscular, índices ponderales y tablas de percentiles de los datos antropométricos útiles en una valoración nutricional. Med Clin 1988;91:223-236. 